\title{
Nanoscale Chiral Recognition Using Field Ion and Field Emission Microscopy
}

\author{
Natalia Gilis $^{1}$, Jai Prakash ${ }^{1,2}$, Cédric Barroo ${ }^{1,3}$ and Thierry Visart de Bocarmé ${ }^{1,3}$ \\ 1. Chemical Physics of Materials and Catalysis, Université libre de Bruxelles, CP243, Brussels, Belgium \\ 2. Department of Chemical Engineering, Indian Institute of Technology (IIT), Kanpur, India \\ 3. Interdisciplinary Center for Nonlinear Phenomena and Complex Systems (CENOLI), Université libre \\ de Bruxelles (ULB), Brussels, Belgium
}

Chirality at surfaces has become an active research area targeting possible applications of enantioselective separation or detection. In this context, significant success has been achieved these past decades by developing new methods for a better understanding of enantiospecific interactions of chiral adsorbate with surfaces $[1,2]$. Here, we propose a promising route to get a fundamental understanding of enantiospecific interaction of chiral molecules on metal surfaces using field emission techniques. These techniques have been chosen for their particular advantage to expose a wide range of structurally different facets in one atomically resolved picture. This diversity enables the screening of interactions between a chemical species and a number of facets during the adsorption process.

In the present study, we envisage the adsorption of alanine on platinum surfaces modelled as sharp tips using field emission techniques. Field Ion Microscopy (FIM) and Field Emission Microscopy (FEM) are used in this study. These two similar techniques allow imaging the surface at the apex of a sharp metallic tip with atomic (for FIM) or nanometric (for FEM) resolution and in real space. They are both based upon the emission of a gas species (FIM) or electrons (FEM) at the surface of a polarized specimen placed in front of a fluorescent screen. The electric field present along the surface induces the formation of the entities that build up the image when projected onto the detector screen. The pattern shows a variety of facets with different structures inherent to the crystal lattice of the sample Figure 1.a. Kinks and steps of the surface are shown on the ball-model of the surface of the tip on Figure 1.c. For FEM the polarity is negative and induces the emission of electrons (Figure 1.b). The brightness will vary in the presence of adsorbed species. The method allows to work with a wide range of temperature and pressure.

In FEM mode, the adsorption of molecules on specific sites would lead to changes in the local work functions associated with the variations in contrasts and brightness on the field-emission patterns. The adsorption of an enantiomer of a well-selected species should show a variation according to the $\mathrm{R}$ or $\mathrm{S}$ orientation of the enantiomorphous facet, and thus lead to a rupture of symmetry of the original FEM micrographs. In order to observe the adsorption pattern of the adsorption, the Pt surface is kept at temperatures between 150 and $300 \mathrm{~K}$, and then exposed to vapors of D or L-alanine. Since alanine is a solid, a solid injection device had to be designed for the microscope. This molecular vapor deposition cell consists of a flask with the organic substance connected to the UHV chamber where the field emitter tip is placed via construction of four folded cross with flexible hose, gauge, turbopump and thermocouple feed-through. The turbo-pump is connected to the flask in order to clean the dosing system by repeated sublimation and evacuation cycles before supplying low amounts of probe molecules to the analysis chamber. The in-situ FEM is recorded with a high-speed video camera. The whole process is also performed in absence of alanine molecules to perform reference experiments (Figure 1.a and 1.b). The subtraction of the results before and after the adsorption provides a net image of the adsorption sites, as shown on Figure 1.d. Our results show a clear preference of the alanine to adsorb on 
chiral facets. Although the $20 \AA$ resolution of the FEM does not allow to unravel the facets with atomic resolution of interest, the net images after exposures to one enantiomer of alanine show the occurrence of an enantioselective adsorption over sector of the same chiral symmetry. The results show that Lalanine has a strong tendency to adsorb on R facets. By symmetry, D-alanine adsorbs on the $\mathrm{S}$ facets. These results are in good agreement with results obtained by DFT calculations [3].

To conclude, we developed a new method to follow the adsorption of chiral material on metallic polycrystalline surface using field ion techniques and demonstrated an enantiospecific adsorption of Land D-alanine adsorption on chiral facets of platinum with R and S symmetries, respectively [4].

\section{References:}

[1] A.J. Gellman, ACS Nano 4 (2010), p. 5.

[2] T. Mallat, E. Orglmeister, A. Baiker, Chem. Rev. 107 (2007), p. 4863.

[3] J.-H. Franke, D.S. Kosov, J. Chem. Phys. 142 (2015), p. 054708.

[4] The authors gratefully thank the Wallonia-Brussels Federation (Action de Recherches Concertées $n^{\circ}$ AUWB 2010-2015/ULB15) and Philippe Wiener - Maurice Anspach Foundation. J.P. thanks the PWMA foundation and ULB for postdoctoral fellowship. C.B. thanks the Fonds de la Recherche Scientifique (F.R.S.-FNRS) for financial support.

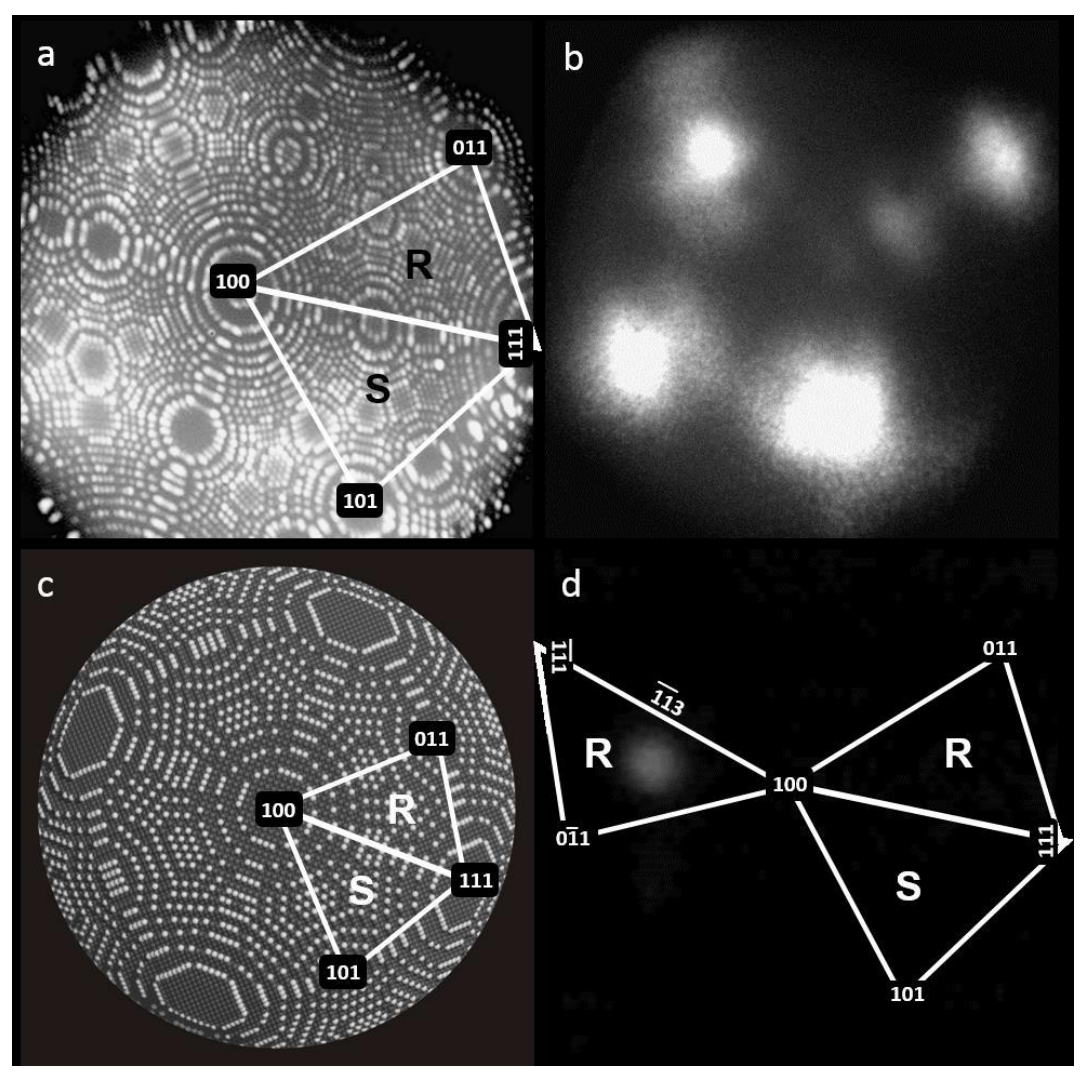

Figure 1. a) Field ion micrograph of a (100)-oriented Pt tip sample (imaging conditions: $\mathrm{T}=50 \mathrm{~K}$, $\mathrm{F}=35 \mathrm{~V} / \mathrm{nm}, \mathrm{P}_{\mathrm{Ne}}=10^{-3} \mathrm{~Pa}$ ) - b) Field electron micrograph of a (100)-oriented Pt tip sample (imaging conditions: $\mathrm{T}=50 \mathrm{~K}, \mathrm{~F}=3 \mathrm{~V} / \mathrm{nm}$ ) - c) Ball model depicting the field ion micrograph - d) Image showing the net adsorption spot of L-alanine on a Pt tip at $300 \mathrm{~K}\left(\mathrm{P}_{\text {alanine }}=3.10^{-4} \mathrm{~Pa}, \mathrm{t}_{\mathrm{ads}}=120 \mathrm{sec}, \mathrm{F}=1.8 \mathrm{~V} / \mathrm{nm}\right)$ showing preferential adsorption on the R-type facets. 